Ti me evol ut i on in vi si bl e light emi ssi on from hi gh- power Ar pul se- nodul at ed i nduct i on ther mal pl asmas

\begin{tabular}{|l|l|}
\hline 著者 & $\begin{array}{l}\text { Tanaka Yasunor i, Tani guchi K, Mur oya } \\
\text { Takaf um, Uesugi Yoshi hi ko }\end{array}$ \\
\hline $\begin{array}{l}\text { j our nal or } \\
\text { publ i cat i on ti t l e }\end{array}$ & I EEE Transact i on on Pl asma Sci ence \\
\hline vol une & 33 \\
\hline number & 2 \\
\hline page r ange & $406-407$ \\
\hline year & $2005-04-01$ \\
\hline URL & ht t p: //hdl . handl e. net /2297/1804 \\
\hline
\end{tabular}




\title{
Time Evolution in Visible Light Emission From High-Power Ar Pulse-Modulated Induction Thermal Plasmas
}

\author{
Yasunori Tanaka, K. Taniguchi, T. Muroya, and Y. Uesugi
}

\begin{abstract}
Transient behavior of a 15-kW Ar pulse-modulated induction thermal plasma (PMITP) at a pressure of $31 \mathrm{kPa}$ was observed using a high-speed video camera. The PMITP method is one of new techniques for controlling temperature and radical density in time-domain in high-power thermal plasmas. The adoption of a high-power metal-oxide-semiconductor field-effect transistor (MOSFET) inverter power supply makes it possible to sustain PMITPs with high power-conversion efficiency. The highspeed video pictures provide two-dimensional, time-dependent radiation intensity distributions from PMITPs, which are useful for understanding an unique transition behavior of PMITPs.
\end{abstract}

Index Terms-High-speed video, inductively coupled plasma, pulse modulation, thermal plasma.

$\mathbf{P}$ ULSE-MODULATED induction thermal plasma (PMITP) method is an unique technique to encounter some disadvantages of steady-state thermal plasmas such as high radiation loss, thermal damage on substrates and grown films, and uncontrollably high enthalpy. Ishigaki et al. first have developed a PMITP system with a static inductive transistor (SIT) operated at a fundamental frequency of $1 \mathrm{MHz}$ [1]. This PMITP is sustained by a periodically modulated coil current. This pulse modulation of the coil current allows thermal plasmas to be under periodical transient state, which has possibilities of controlling thermal plasma temperature and radical density in time domain, and of introducing chemically and thermally nonequilibrium effects on high-power plasmas. Our group has also developed such a PMITP system with a metal-oxide-semiconductor field-effect transistor (MOSFET) operated at $450 \mathrm{kHz}$ [2], [3], and then has confirmed the thermal plasma temperature controlled by modulation of the coil-current in various working gases [2]. In our previous works [2], spectroscopic observation was carried out to obtain the transient behavior of the radiation intensity of $\mathrm{Ar}$ spectral lines and that of Ar excitation temperature at specified points [2]. However, it is crucial to understand the multidimensional unique behavior of PMITPs, which is governed by the coupling of the fluid flow and electromagnetic fields. The high-speed video capture is useful for this purpose. This report addresses the two-dimensional (2-D) transient behavior of such Ar PMITPs captured with a high-speed video camera.

Fig. 1 illustrates a schematic diagram of the plasma torch for PMITPs. The detailed configuration was already reported in [2].

Manuscript received June 2, 2004.

The authors are with the Department of Electrical and Electronic Engineering, Kanazawa Univeristy, Kanazawa 920-8667, Japan (e-mail: tanaka@ ec.t.kanazawa-u.ac.jp).

Digital Object Identifier 10.1109/TPS.2005.844994

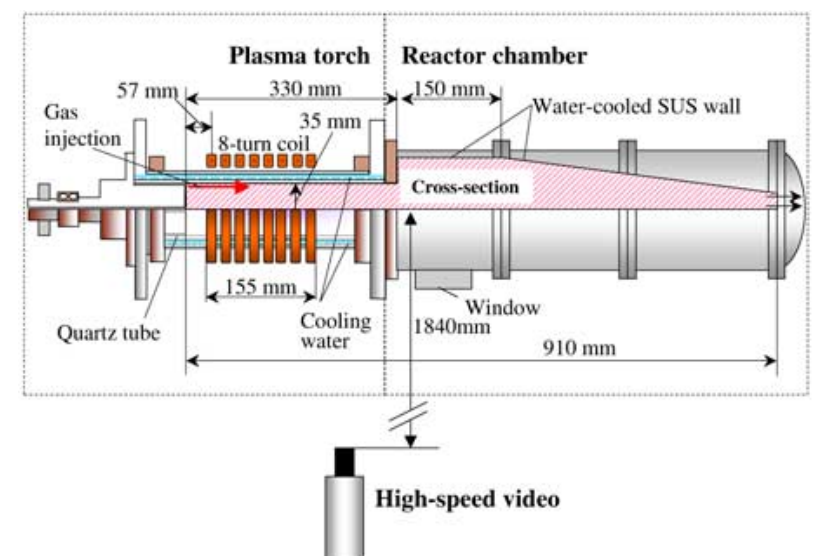

Fig. 1. Schematic diagram of plasma torch and reaction chamber. (a) (b) (c) (d) (e) (f) (g) (h)

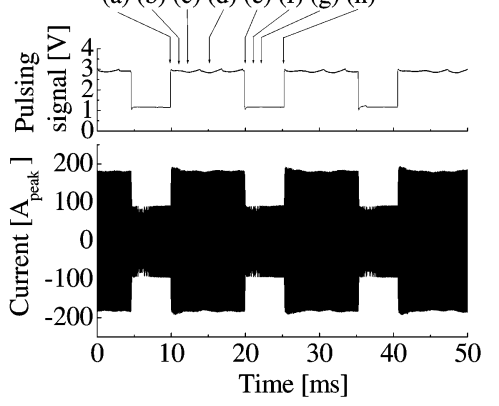

Fig. 2. Pulsing signal and coil-current waveform of Ar PMITP at a pressure of $31 \mathrm{kPa}$ at a time-averaged power of $15 \mathrm{~kW}$. On-time is $10 \mathrm{~ms}$ and off-time is $5 \mathrm{~ms}$. The shimmer current level is $50 \%$.

The Ar gas flow rate was set to $100 \mathrm{l} / \mathrm{min}\left(=1.67 \times 10^{-3} \mathrm{~m}^{3} / \mathrm{s}\right)$. In this experiment, an observation window is located at around $190 \mathrm{~mm}$ from the coil-end on the reaction chamber. Pressure inside the reaction chamber was controlled to be at $31 \mathrm{kPa}$ (= 230 torr). The time-averaged input power to the MOSFET inverter power supply was fixed at $15 \mathrm{~kW}$ in this experiment. The quantity "on-time," which is defined as a time duration with higher current amplitude level, was set to $10 \mathrm{~ms}$, and "off-time," which is a time duration with lower current amplitude level, was $5 \mathrm{~ms}$ [2]. A high-speed video camera with a complementary metal-oxide semiconductor (CMOS) optical array was set up at $1840 \mathrm{~mm}$ away from the plasma torch. The CMOS has a sensitivity for visible light between $400-800 \mathrm{~nm}$. The exposure time of this high-speed video camera was set to $10 \mu \mathrm{s}$, whereas a frame rate was independently set to 3200 frame/s. 

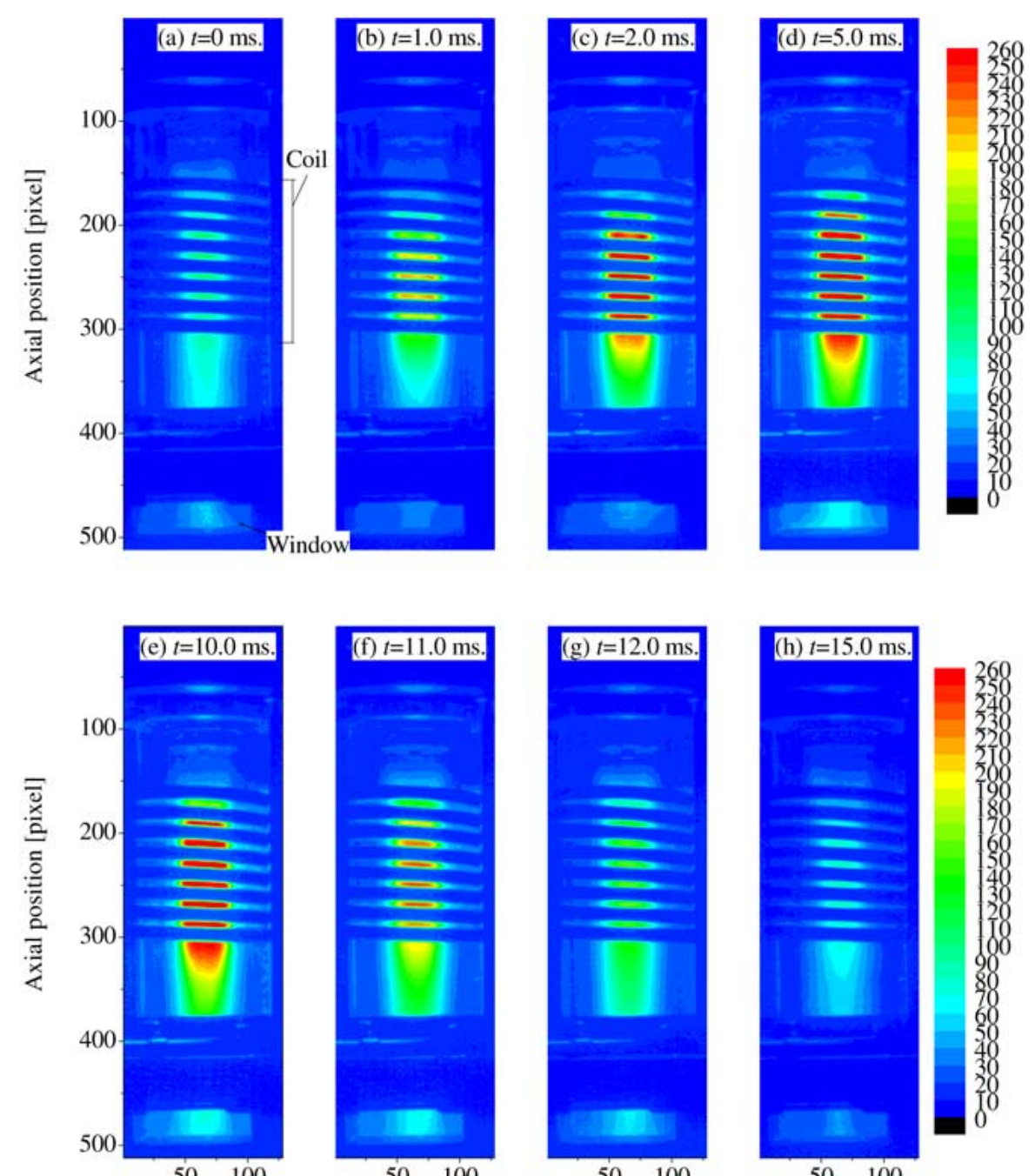

Radial position [pixel]

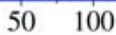

Fig. 3. Time evolution in visible light emission from Ar PMITP at a pressure of $31 \mathrm{kPa}$ at a time-averaged power of $15 \mathrm{~kW}$. On-time is $10 \mathrm{~ms}$ and off-time is $5 \mathrm{~ms}$. Shimmer current level is $50 \%$.

Fig. 2 shows the pulsing signal for controlling coil current and the coil-current waveform of an Ar PMITP. The amplitude of the coil current is confirmed to be modulated into an almost square waveform corresponding directly to the pulsing signal. Fig. 3 depicts a transition of a 2-D light intensity distribution from the Ar PMITP. In this figure, the light intensity is denoted from 0 to 256 levels with color. Each figure (a)-(h) corresponds to an image captured at a time point as denoted in Fig. 2. Just after on-operation, as seen in Fig. 3(a), the light emission region is still small and has lower light intensity. From the window located at around $190 \mathrm{~mm}$ from the coil-end, the light emission region can be seen. At $1.0 \mathrm{~ms}$ after the on-operation, as seen in Fig. 3(b), a plasma grows up mainly inside the coil-region. At this time, the light intensity around the window decreases. From $t=1.0 \mathrm{~ms}$ to $5.0 \mathrm{~ms}$, the light intensity of the PMITP increases, the light emission region expands axially and radially. At $5.0 \mathrm{~ms}$, a gas flow with a high light intensity from the upstream portion reaches to the window position. From 5.0 to $10.0 \mathrm{~ms}$, the light intensity from the PMITP hardly changes, which means that the PMITP achieves almost a steady condi- tion at $5.0 \mathrm{~ms}$ after on-operation. At $1.0 \mathrm{~ms}$ after "off-operation," which is denoted by $t=11.0 \mathrm{~ms}$ in Fig. 3 (f), the light intensity from the PMITP quickly decays, especially inside the coil-region. From $t=11.0 \mathrm{~ms}$ to $12.0 \mathrm{~ms}$, the light intensity mainly inside the coil-region decays, whereas the light emission region remains as it is. This light intensity finally decreases from $t=12.0$ to $15.0 \mathrm{~ms}$, which leads the plasma to be small. The above transition tendency depends remarkably on gas kind used in the plasma torch.

\section{REFERENCES}

[1] T. Ishigaki, F. Xiaobao, T. Sakuta, T. Banjo, and Y. Shibuya, "Generation of pulse-modulated induction thermal plasma at atmospheric pressure," Appl. Phys. Lett., vol. 71, pp. 3787-3789, 1997.

[2] Y. Tanaka and T. Sakuta, "Temperature control of Ar induction thermal plasma with diatomic molecular gases by pulse-amplitude modulation of coil-current," Plasma Sources Sci. Technol., vol. 12, pp. 69-77, 2003.

[3] M. M. Hossain, Y. Tanaka, and T. Sakuta, "Dynamic responses of Ar- $\mathrm{CO}_{2}$ and $\mathrm{Ar}-\mathrm{N}_{2}$ induction thermal plasmas in pulse modulation approach: A numerical analysis," Thin Solid Film, vol. 435, pp. 19-26, 2003. 